\title{
Amiloidosis en Didelphis marsupialis infectado experimentalmente con Leishmania chagasi
}

\author{
Diana Milena Roa ${ }^{1}$, Ladys Sarmiento ${ }^{2}$, Gerzaín Rodríguez ${ }^{1,3}$ \\ ${ }^{1}$ Laboratorio de Patología, Instituto Nacional de Salud, Bogotá, D.C., Colombia. \\ 2 Unidad de Microscopía y Análisis de Imágenes, Instituto Nacional de Salud, Bogotá, D.C., Colombia. \\ ${ }^{3}$ Departamento de Patología, Facultad de Medicina, Universidad Nacional de Colombia, Bogotá, D.C., \\ Colombia.
}

Un Didelphis marsupialis macho capturado en Teruel, Huila, se inoculó intraperitonealmente con $1 \times 10^{6}$ promastigotes de Leishmania chagasi (MHOM/CO/84/CL044B). El animal desarrolló leishmaniosis visceral con abundantes amastigotes fagocitados en las células de Kupffer y en los macrófagos esplénicos; murió 5 semanas después de la inoculación. La autopsia reveló amplios depósitos de amiloide en el hígado y en el bazo, demostrados por sus características tintoriales y ultraestructurales. La consideramos como una amiloidosis secundaria a la infección experimental, que ocurrió en el transcurso de 5 semanas luego de inoculado el animal.

Palabras clave: Didelphis marsupialis, leishmaniosis visceral, amiloidosis secundaria.

Amyloidosis in Didelphis marsupialis experimentally infected with Leishmania chagasi

A male opossum, Didelphis marsupialis, captured in Teruel (Huila), Colombia, was inoculated intraperitoneally with $1 \times 10^{6}$ promastigotes of Leishmania chagasi (MHOM/CO/84/CL044B). The animal died 5 weeks after inoculation. Autopsy revealed signs of visceral leishmaniasis along with amastigote parasite form in Kupffer cells and spleen macrophages. Amyloid deposits in liver and spleen were demonstrated by histological staining and electron microscopy. The rapid death was considered a consequence of a secondary, reactive amyloidosis.

Key words: Didelphis marsupialis, visceral leishmaniasis, secondary amyloidosis.

Un Didelphis marsupialis macho (fara, chucha, zarigüeya) fue capturado por personal del Instituto Nacional de Salud mediante el empleo de trampas en Teruel, Huila; el animal se inoculó intra-peritonealmente con $1 \times 10^{6}$ promastigotes de Leishmania chagasi (MHOM/CO/84/CL044B), cepa procedente de un paciente de Pulí, Cundinamarca; el animal falleció 5 semanas después de la inoculación. Las imágenes ilustran cortes de hígado del animal.

En la figura 1a, coloreada con hematoxilinaeosina (HE), se ven abundantes amastigotes fagocitados por las células de Kupffer, parásitos

Correspondencia:

Gerzaín Rodríguez, Laboratorio de Patología, Instituto Nacional de Salud, Avenida Calle 26 No.51-60, Bogotá, D.C., Colombia;

teléfono 220 7700; grodriguez@hemagogus.ins.gov.co

Recibido: 20/06/02; aceptado: 23/08/02 que se aprecian como estructuras granulares (flechas); es también llamativa la atrofia importante de los hepatocitos que están reducidos a cadenas tenues por la presencia de un material eosinófilo, homogéneo, que delimita los sinusoides que se ven llenos de eritrocitos. Este material se interpretó como amiloide, lo cual se confirmó con la coloración de rojo Congo que lo tiñó de color caoba (figura 1b) y fue birrefringente con luz polarizada; además, fue fluorescente con la tioflavina $T$, técnica con la que el amiloide aparece en forma de cintas verdes (figura 1c). En esta misma figura, el material autofluorescente amarillento, particulado, corresponde a los eritrocitos dentro de los sinusoides. Por microscopía electrónica se observaron abundantes amastigotes fagocitados por las células de Kupffer (figura 2a) y el amiloide mostró aspecto fibrilar con haces de filamentos en forma de agujas entrelazadas, de 7 a $10 \mathrm{~nm}$ 


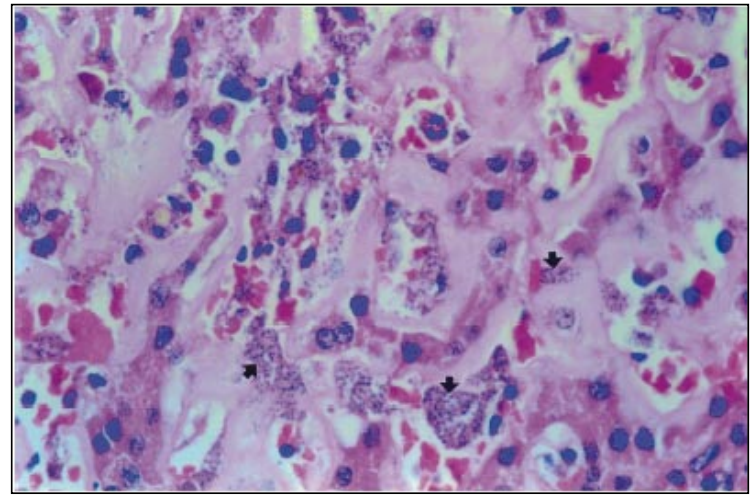

Figura 1a. Hígado: descripción en el texto. HE, 80X.
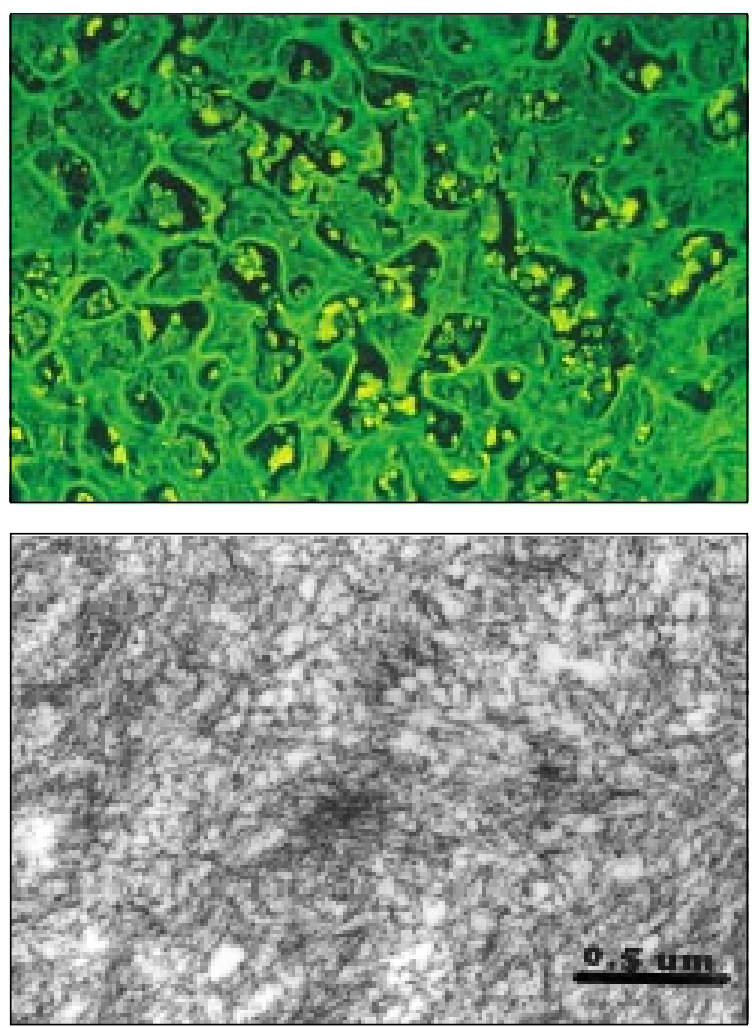

Figura 2a. Célula de Kupffer en el centro, con numerosos amastigotes fagocitados. El material denso fibrilar $(A)$ es amiloide. Escala: 3,6 $\mu \mathrm{m}$.

de diámetro, el típico aspecto ultraestructural para todas las formas de amiloide (figura $2 b$ ).

Otros órganos del animal estudiados fueron el bazo y el pulmón. En el bazo también había depósitos de amiloide perivasculares, menos notorios que en el hígado, junto con abundantes amastigotes fagocitados por los macrófagos. En

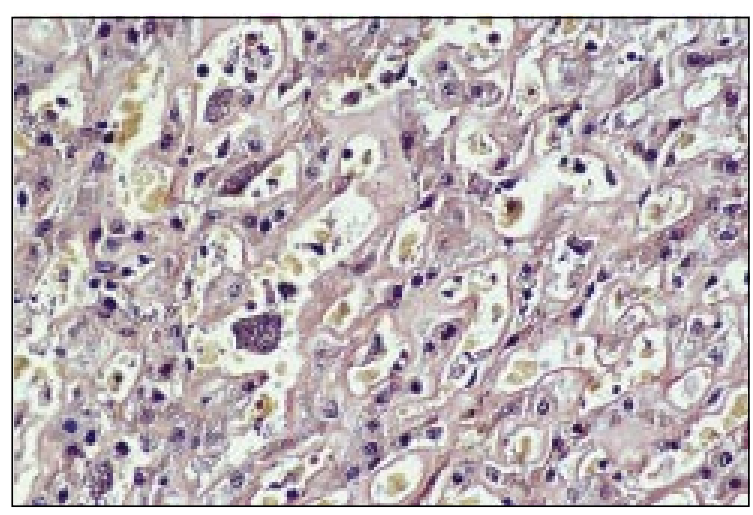

Figura 1b. Hígado: descripción en el texto. Rojo Congo, $80 \mathrm{X}$.

Figura 1c. Hígado: descripción en el texto. Tioflavina $\mathrm{T}$ y microscopía con luz ultravioleta, 80X.

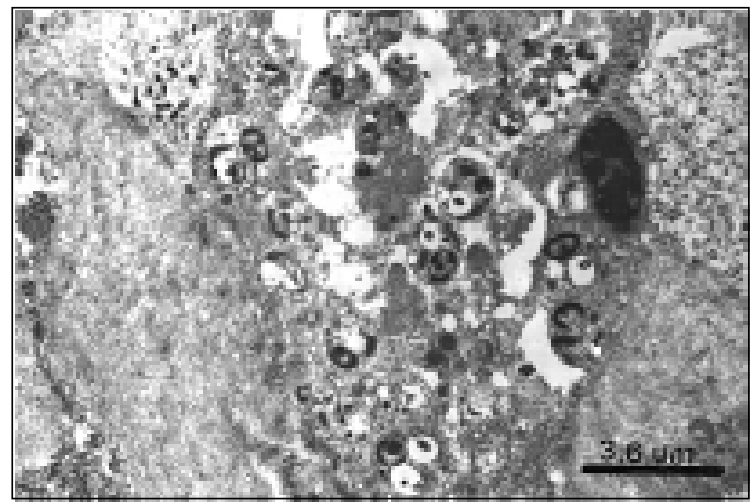

Figura 2b. Aspecto fibrilar típico del amiloide, con mayor resolución. Escala: 0,5 $\mu \mathrm{m}$.

el pulmón no se vieron depósitos de amiloide ni infiltrados inflamatorios con amastigotes.

\section{Comentario}

Los Didelphis marsupialis son reservorios de varios agentes infecciosos como los que ocasionan la fiebre amarilla $(1,2)$, la leishmaniosis visceral y la enfermedad de Chagas (3-5); 
presentan una alta tasa de infección natural por los parásitos de la familia Trypanosomatide, organismos que se detectan en su sangre (5).

Las amiloidosis incluyen un grupo de entidades en varios órganos y tejidos, caracterizadas por el depósito extracelular de proteínas de estructura fibrilar, insolubles, de morfología y características tintoriales semejantes, pero de composición química distinta $(6,7)$; el depósito puede ser focal o multisistémico, generalizado; en este caso, se altera la función de órganos vitales como el hígado, el riñón o los vasos sanguíneos, comprimidos o reemplazados por el amiloide, lo cual conduce a la muerte del hombre o del animal afectado $(8,9)$.

El amiloide está formado por dos elementos bien definidos: un componente fibrilar y una unidad pentagonal o componente $\mathrm{P}$; ambos han sido identificados en todas las amiloidosis y se ha demostrado que el componente $P$ es idéntico en todas ellas, mientras que el componente fibrilar es característico de cada tipo de amiloidosis, según la proteína que lo origine $(6,7)$.

La clasificación de la amiloidosis se basa en el tipo de proteína precursora que forma el amiloide; los principales tipos son los siguientes $(7,8)$ :

AL: el precursor de esta sustancia amiloide es la fracción variable o $\mathrm{N}$-terminal de las cadenas livianas de las inmunoglobulinas; este depósito se presenta en las amiloidosis primarias sistémicas.

AA: el precursor es la proteína sérica $A$, presente en el suero normal y en diferentes estados inflamatorios o tumorales; origina las amiloidosis secundarias a inflamaciones crónicas y tumores.

AFp: su precursor es la prealbúmina sérica, amiloidosis que se asocia con la polineuropatía familiar.

AS: denominación para el amiloide senil, que se localiza en el aparato cardiovascular (ASc) y en el cerebro (ASb).

AEp: amiloide endocrino de los islotes pancreáticos. Su precursor es la insulina.

AEt: amiloide endocrino del carcinoma medular del tiroides, cuyo precursor es la tirocalcitonina.
AD: amiloide de la piel, localizado en la dermis papilar; la proteína precursora es la queratina.

Las amiloidosis más frecuentes en humanos en Colombia y en muchos países en desarrollo son las secundarias o reactivas, llamadas así porque se desencadenan por la inflamación crónica persistente en enfermedades como la tuberculosis o la lepra, en las cuales la proteína precursora es la SAA o proteína amiloide A del suero, producida en el hígado como respuesta a la inflamación crónica $(8,9)$. En los países desarrollados, la amiloidosis más frecuente en humanos es la primaria sistémica, cuyos precursores son las cadenas livianas de inmunoglobulinas (10).

Las coloraciones tradicionales para el amiloide como el rojo Congo, la tioflavina $T$ y otras dispendiosas o poco específicas $(6,11)$ están siendo reemplazadas por métodos inmunohistoquímicos que demuestran la presencia del componente AP y la proteína específica de cada tipo de amiloide $(6,7)$.

Aun cuando no demostramos la naturaleza química del amiloide del Didelphis marsupialis, pensamos que es secundaria a la infección leishmaniósica y que se depositó en un tiempo muy corto, aunque no tenemos una prueba incontrovertible de esta afirmación porque desconocemos el estado de salud previo del animal. No obstante, este depósito de amiloide no ha sido antes observado en Didelphis infectados naturalmente con diversos agentes animales que han sido estudiados en el Laboratorio de Patología del INS. Por otra parte, otros animales experimentales como el hámster, desarrollan amiloidosis secundaria sistémica con la leishmaniosis experimental $(12,13)$. En los humanos deben transcurrir varios años con la infección crónica para que se deposite el amiloide (9); lo mismo ocurre con los caballos inoculados repetidamente para producir sueros antirrábicos o antiofídicos, los cuales desarrollan amiloidosis secundaria sistémica después de varios años de ser inoculados con los inmunógenos correspondientes (Laboratorio de Patología, INS, observaciones sin publicar).

La amiloidosis secundaria ocurre con regularidad en modelos experimentales de animales que reciben inyecciones reiteradas de antígenos, 
como en los hámsteres $(12,13)$, ratones (14) y perros con leishmaniosis visceral (15); los depósitos se ven en el hígado, el bazo, las glándulas suprarrenales, el riñón y los testículos $(16,17)$.

En conclusión, un Didelphis marsupialis con infección experimental por L. chagasi desarrolló amplios depósitos viscerales de amiloide en el hígado y en el bazo que se demostraron por sus características tintoriales y ultraestructurales; consideramos esta amiloidosis como secundaria a la infección experimental con este parásito; estos eventos se desarrollaron en 5 semanas.

\section{Referencias}

1. Boshell J. Informe sobre la fiebre amarilla silvestre en la región del Meta, desde julio de 1934 hasta diciembre de 1936. Rev Fac Med 1938;6:407-27.

2. Gast-Galvis A. Historia de la fiebre amarilla en Colombia. Bogotá: Instituto Nacional de Salud; 1982.

3. Corredor A, Gallego JF, Tesh RB, Peláez D, Díaz A, Montilla M, et al. Didelphis marsupialis, an apparent wild reservoir of Leishmania donovani chagasi in Colombia, South America. Trans R Soc Trop Med Hyg 1989;83:195

4. Travi BL, Osorio Y, Guarín N, Cadena H. Leishmania (Leishmania) chagasi: clinical and parasitological observations in experimentally infected Didelphis marsupialis, reservoir of New World visceral leishmaniasis. Exp Parasitol 1998;88:73-5.

5. Travi BL, Jaramillo C, Montoya J, Segura I, Zea A, Goncalves A, et al. Didelphis marsupialis, an important reservoir of Trypanosoma (Schizotrypanum) cruzi and Leishmania (Leishmania) chagasi in Colombia. Am J Trop Med Hyg 1994;50:557-65.

6. Muñoz J. Diagnóstico y seguimiento de la amiloidosis. Rev Esp Reumatol 1996;23:21-5.
7. ABC Tu Salud. Amiloidosis. 2001; (2 pantallas). Disponible en: URL: http://www.abctusalud.com.

8. Panqueva C, Rodríguez G. Amiloidosis sistémica en autopsias. Revisión de la casuística del Hospital San Juan de Dios de Bogotá. Acta Med Col 1986;11:87-91.

9. Rodríguez G, Berrío J, Sarmiento L. La lepra y el riñón. Biomédica 1999;19:45-55.

10. Kyle R, Bayrd E. Amyloidosis: review of 236 cases. Medicine 1975;54:271-98.

11. Sipe JD, Cohen AS. Review: history of the amyloid fibril. J Struct Biol 2000;130:88-98.

12. González J, Gallego E, Castaño M, Rueda A. Testicular amyloidosis in hamsters experimentally infected with Leishmania donovani. Br J Exp Pathol 1983;64:518-23.

13. Sartori A, Oliveira AV, Roque-Barreira MC, Rossi MA, Neto-Campos A. Immune complex glomerulo-nephritis in experimental Kala-azar. Parasite Immunol 1987;9:93103.

14. Barbosa-Santos E, Goncalves-Da Costa S, Alencar A. Amiloidosis in experimental tegumentary leishmaniasis in mice. Am J Trop Med Hyg 1984;33: 1270-1.

15. Poli A, Abramo F, Mancianti F, Nigro M, Pieri S, Bionda A. Renal involvement in canine leishmaniasis. A light-microscopic, inmunohistochemical and electronmicroscopic study. Nephron 1991;57:444-52.

16. Kennedy J, Anderson JD. The effect of treatment of the associated disease on the development of amyloidosis in the experimental animal. J Pathol 1983; $141: 11-5$

17. González J, Gallego E, Castaño M, Rueda A. Testicular amyloidosis in hamsters experimentally infected with Leishmania donovani. $\mathrm{Br} \mathrm{J}$ Exp Pathol 1983; 64:518-23. 


\section{Fe de erratas}

\section{Amiloidosis en Didelphis marsupialis infectado experimentalmente con Leishmania chagasi}

Diana Milena Roa ${ }^{1}$, Ladys Sarmiento ${ }^{2}$, Gerzaín Rodríguez ${ }^{1,3}$

Véase figura $2 a$ y figura $2 b$

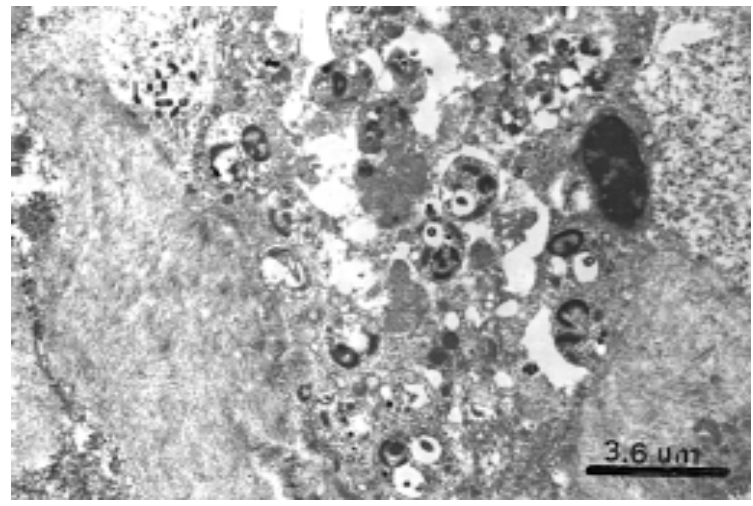

Figura 2a. Célula de Kupffer en el centro, con numerosos amastigotes fagocitados. El material denso fibrilar (A) es amiloide. Escala: 3,6 $\mu \mathrm{m}$.

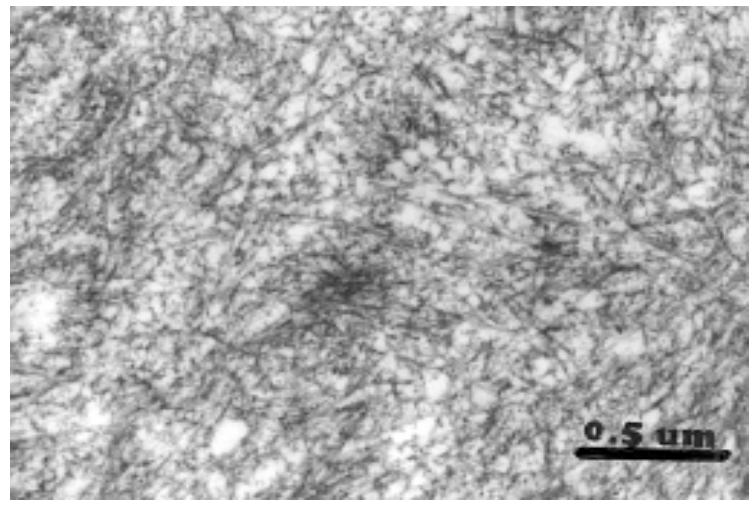

Figura 2b. Aspecto fibrilar típico del amiloide, con mayor resolución. Escala: $0,5 \mu \mathrm{m}$.

\footnotetext{
${ }^{1}$ Laboratorio de Patología, Instituto Nacional de Salud, Bogotá, D.C., Colombia.

2 Unidad de Microscopía y Análisis de Imágenes, Instituto Nacional de Salud, Bogotá, D.C., Colombia.

${ }^{3}$ Departamento de Patología, Facultad de Medicina, Universidad Nacional de Colombia, Bogotá, D.C., Colombia.
} 


\title{
Insuficiencia suprarrenal secundaria a paracoccidioidomicosis
}

\author{
José M. Oñate ${ }^{1}$, Angela María Tobón ${ }^{2}$, Angela Restrepo ${ }^{2}$
}

\section{Véase figura 1}

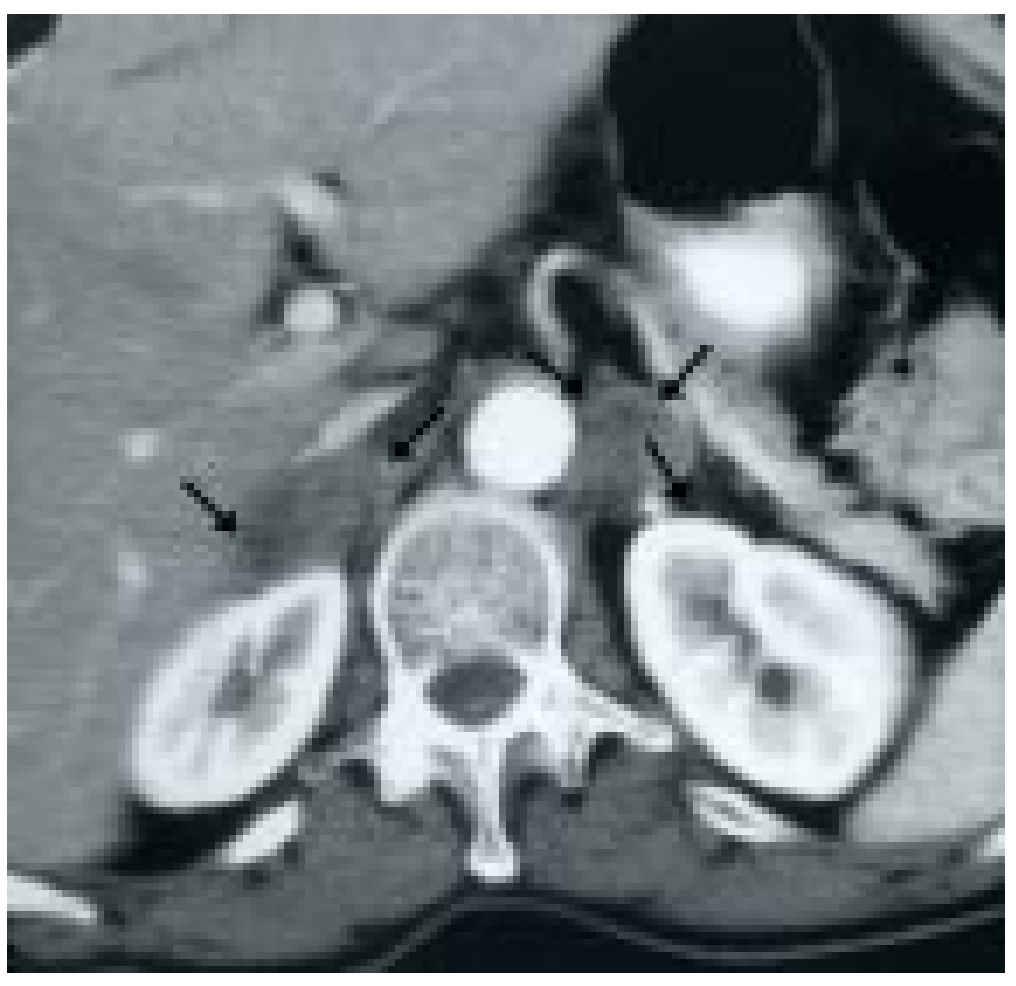

Figura 1. Tomografía computarizada de las glándulas suprarrenales. Se observan lesiones hipodensas que comprometen las glándulas suprarrenales de manera bilateral (flechas largas). Nótese la presencia de calcificaciones en la glándula suprarrenal izquierda (flecha corta).

\footnotetext{
1 Universidad del Valle, Cali, Colombia.

2 Grupo de Micología Médica, Corporación para Investigaciones Biológicas, Medellín, Colombia.
} 